\title{
Sex ratio in Multiple Sclerosis mortality over 65 years; An Age-Period-Cohort Analysis in Norway
}

\author{
Ola Nakken ${ }^{1,2}$, Jonas Christoffer Lindstrøm ${ }^{1,3}$, Trygve Holmøy ${ }^{1,2}$ \\ ${ }^{1}$ Institute of Clinical Medicine, Campus Ahus, University of Oslo, Oslo, Norway \\ ${ }^{2}$ Department of Neurology, Akershus University Hospital, Lørenskog, Norway \\ ${ }^{3}$ Health Services Research Unit, Akershus University Hospital, Lørenskog, Norway
}

Correspondance to: Ola Nakken, email: ola.nakken@medisin.uio.no, telephone +4797659796, fax +4767968860

\section{Acknowledgements}

We are grateful for data supplied by the Norwegian Cause of Death Registry

\begin{abstract}
Increasing female: male ratio in multiple sclerosis (MS) has been assigned to cohort effects, with females in more recent birth cohorts possibly being more exposed or vulnerable to environmental risk factors than males. We collected MS mortality data in Norway from 1951 to 2015 from The Norwegian Cause of Death registry. Age-Period-Cohort analysis was conducted using log-linear Poisson models, including sex interaction terms. MS was registered as the underlying, contributing or direct cause in 6060 deaths. MS associated mortality remained stable with a slight preponderance among males until after 1980, and have since increased preferentially among females. Throughout the study period the mean annual increase was $1.25 \%$ for females and $0.3 \%$ for males $(p<0.0001)$. Age-period-cohort analysis revealed limited evidence of cohort effects for the gender differences; the best fitting model only included gender-age and gender-period interaction terms. The period effect evened out for males in the last three decades but increased for females, especially among the oldest age-groups. In conclusion, the increased female: male mortality ratio in MS associated mortality is driven mainly by increased mortality among females in the three last decades, particularly in the older age groups. It is best explained by disproportional period effects, providing evidence of time-varying external factors including improved access to diagnosis among females.
\end{abstract}

\section{Keywords}

Multiple Sclerosis, sex ratio, neuro-epidemiology, mortality, age-period-cohort model 


\section{Introduction}

The female/male sex ratio may serve as a robust epidemiological marker, to some degree bypassing the methodological concerns apparent when comparing incidence and prevalence data from different times and regions [1]. An increase in sex ratio among more recently born multiple sclerosis (MS) patients is possibly determined by a preferential increase in women with MS in many [2-6], but not all geographical areas [7]. The relatively rapid increase among females has been attributed to environmental impact preferentially affecting women [1], also in Norway [6]. However, a disproportionate improvement in case ascertainment over time, with gradually more equal access to diagnosis for women and men, could also possibly contribute to an increase in the observed sex ratio.

Age-period-cohort models provide a tool for interpreting temporal changes in epidemiological rates and ratios [8]. Age effects reflect how many years an individual has been alive at the registration. Period effects reflect the time-varying influence that affect all cohorts equally as they age, and typically represent external factors such as changes in case ascertainment. Cohort effects reflect variations in disease between birth cohorts, and are associated with long-term exposures affecting different generations being exposed to different risks. An extended age-period-cohort analysis provides opportunities to assess whether changes in sex ratio are due to interactions between the effect of gender and the three time effects.

The aim of this study was to assess long-term changes in sex ratio in MS associated mortality in Norway, and to evaluate the potential effect of age, period and cohort on these trends.

\section{Materials and Methods}

The Norwegian Cause of Death Registry (NCoDR) collects, processes and evaluates all information on all death certificates in Norway. Digitalized files are available from 1951 onwards. Cause of death is typically divided into the direct, contributing or underlying. Each cause is coded according to the international classification of diseases (ICD). The following ICD versions have been used; 1951-1957: ICD 6, 1958-1968: ICD 7, 1969-1985: ICD 8, 1986-1995: ICD 9, 1996 onwards: ICD 10. We searched all death certificates from 1951 to 2015 containing codes corresponding to MS at any level of cause of death, and also for underlying cause separately. The following codes were selected; ICD 6 and 7: 345, ICD 8 and 9: 340, ICD 10: G35. Data was aggregated according to age ( 5 year bins), period of death ( 5 year bins) and gender. Cohorts were calculated by extracting age from period of death, providing 22 overlapping 10-year birth cohorts from 1871-1880 to 1976-1985. General population data corresponding to each period and age-group were obtained from Statistics Norway.

We used Poisson regression to test for longitudinal differences in gender-specific trends. The following model was specified;

$$
\mu=\text { Personyears } \times \mathrm{e}^{\beta_{0}+\beta_{1} \text { Sex }+\beta_{2} \text { Period }+\beta_{3} \text { Sex } \times \text { Period }}
$$

where $\mu$ is the expected number of MS deaths for each category of sex and period ( 5 year periods coded on a continuous scale). The regression coefficient $\beta_{3}$ is interpreted as the annual difference between gender specific rates. For graphical purposes, gender-specific rates were standardized, using the Norwegian 2011-2015 population as reference. The same procedure was used for all MS related mortality and for cases with MS recorded as underlying cause of death.

We performed an age-period-cohort analysis as described by Carstensen [9]. Because of the linear dependence between age, period and cohort effects (cohort=period-age), simultaneous estimation of all three linear effects is not feasible without arbitrary additional model constraints. We chose 1970 as reference period and 1925 as 
reference cohort. For the main analysis, we constrained the cohort effect to be zero on average, with zero slope. In one sensitivity analysis we shifted the constraints over to the period effect. In addition to age, period and cohort, sex was introduced as a main effect. To identify which time effects that influenced changes in sex ratio, multiplicative age-sex, period-sex and cohort-sex interaction effects were also added in consecutive models. The model selection in APC analysis is data driven, where goodness of fit from the different models are compared using likelihood ratio tests, and evidence for more complex models is greater when the p-value is low. As pvalues cannot be calculated in non-nested models, Akaike's Information Criterion (AIC) provides a tool for evaluating goodness of fit, with lower AIC-value indicating less information loss and better model fit [10]. All analyses were carried out using R software (http://www.R-project.org).

\section{Results}

Between 1951-2015, 6060 (55.3\% women) deaths were coded with MS in the death certificate, including 4189 (55.1\% women) with MS as an underlying cause of death. Age-standardized mortality rates for males and females are given in Fig1. MS related mortality was marginally higher among men until 1980, but from there after increased preferentially among females. Mean female/male sex ratio was 0.95:1 at the beginning of our observation period (1951-1955) and ended at 1.8:1 (2011-2015). From the poisson model, $\beta_{3}$ for interaction between period and gender was estimated to 0.0093 (95\% CI $0.0066-0.0121)$, corresponding to an annual increase among women of $1.25 \%$ and $0.3 \%$ among men $(\mathrm{p}<0.001)$. Restricting the analysis to MS as underlying cause of death showed similar interaction between period and gender $\left(\beta_{3}=0.0073, p<0.001\right.$; Supplementary Figure 1). Analyses of direct and contributing causes of death were not possible due to changes in recording and reporting practice.

Age-specific rates by cohorts increased among the elderly but decreased among the younger age groups (Figure 2). This discrepancy was most obvious among females. A similar trend was observed for period of death (Figure 3 ), where age-specific rates were rather stable for females up until 1980, after which they diverged. While the rates for younger age-groups also dropped among males, the increase among the older age-groups was less obvious (Figure 3).

To more formally assess the relative impact of the main time effects on the increasing sex ratio, we next compared age-period-cohort models with all possible interactions between gender and time effects. Both the model including gender-cohort interaction $(\mathrm{p}<0.001)$ and gender-period interaction $(\mathrm{p}<0.001)$ fitted our data significantly better than the model only including the main effects, while the model only including gender-age interaction did not $(\mathrm{p}=0.10)$ (supplementary Table 1). Comparing all candidate models, AIC indicated that the best model included gender-period and gender-age interaction, but not gender-cohort interaction.

Predicted period and birth cohort effects for males relative to females are displayed in Figure 4A and 4B. For period effects, there was a steady increase among females from late 1970-ies to 2015, while the relative period effect for males declined from the same breaking point (Figure 4A). Cohort effects will be deviations from the rate predicted by the age-period combination. These effects demonstrate increasing risk for both genders in consecutive cohorts up until 1940-ies, with a subsequent possible decline. The gender-cohort interaction is less obvious than the gender-period interaction with this parameterization (Figure 4B). When shifting the constraints over to the period effect, the relative cohort effect for males seemed to decrease somewhat, particularly before 1940. Otherwise, the qualitative interpretation of both period and cohort effects remains the same (supplementary Figure 2A and 2B). 


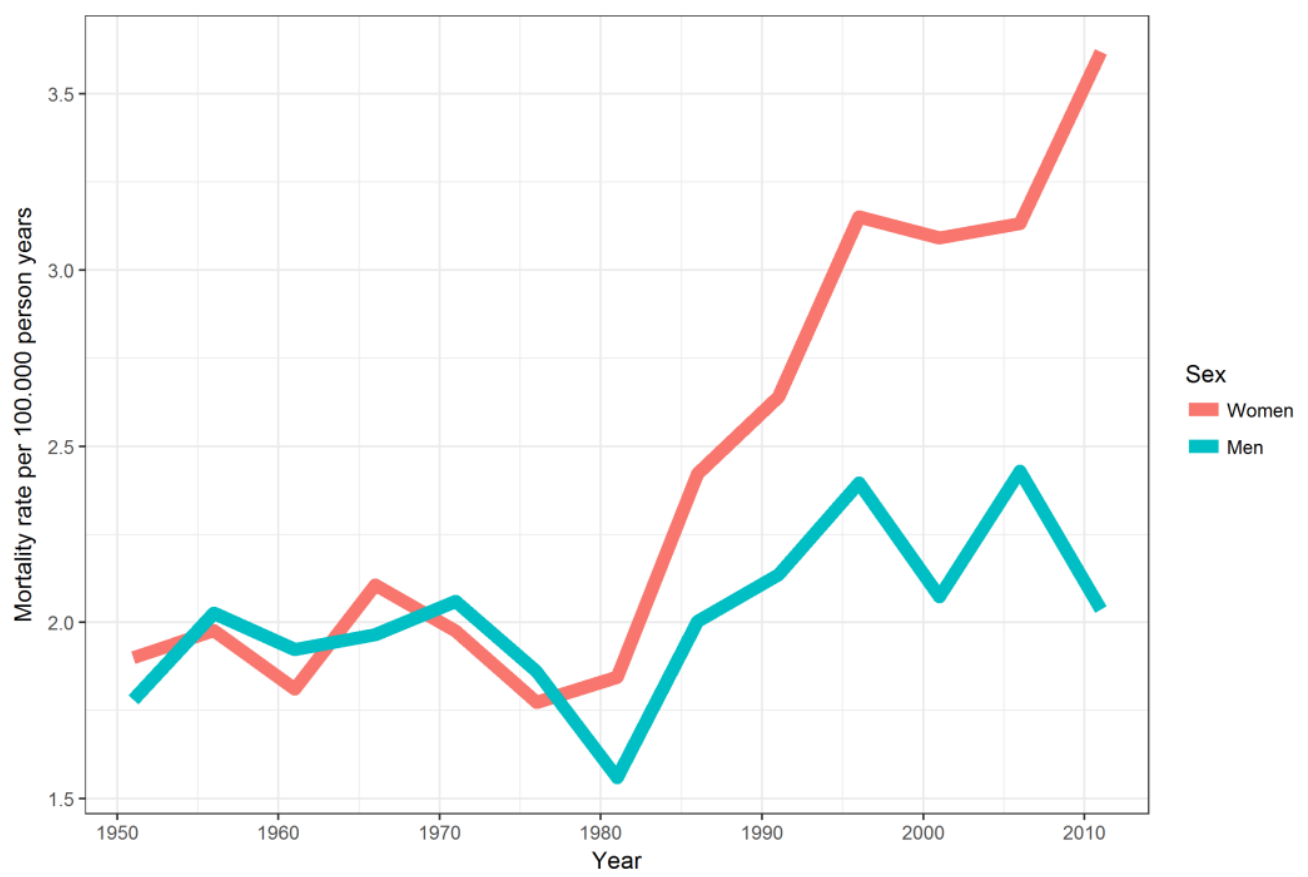

Fig.1 Multiple Sclerosis mortality in Norway 1951-2015 by sex and period of death. Rates are standardized using the 2011-2015 Norwegian population as reference.

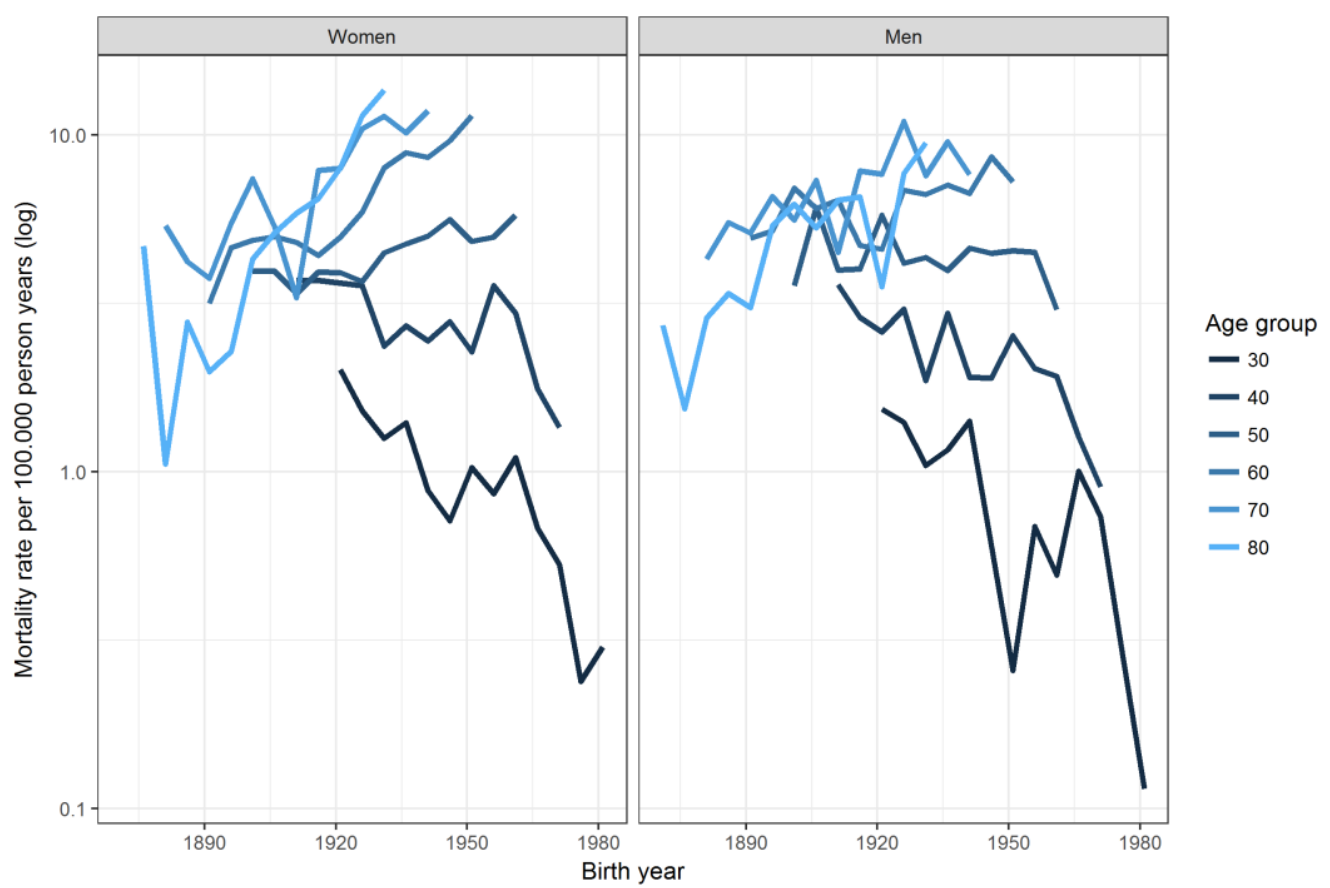

Fig.2 Multiple Sclerosis mortality in Norway 1951-2015 in different age groups by birth cohort. 


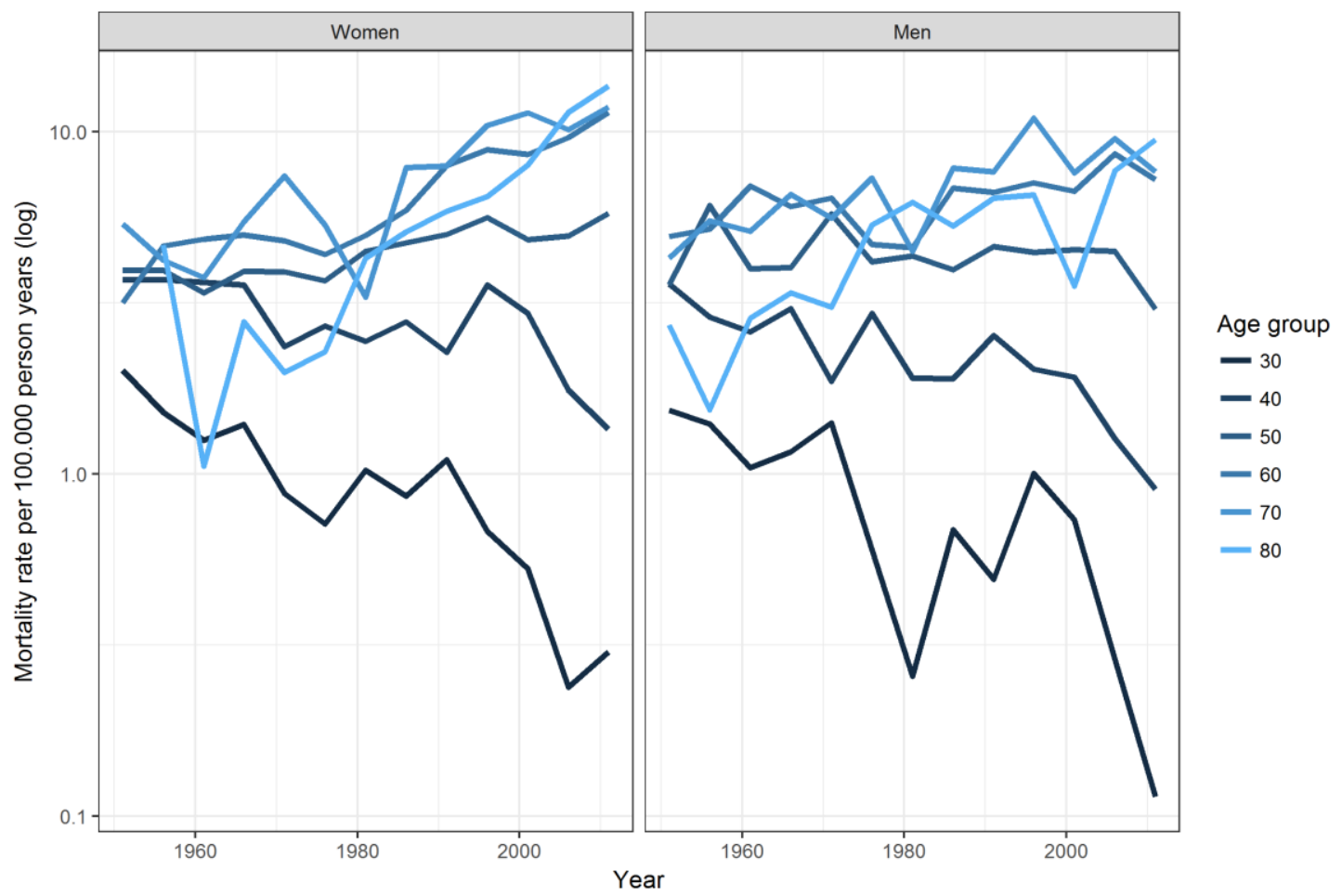

Fig.3 Multiple Sclerosis mortality in Norway 1951-2015 in different age groups by period of death.
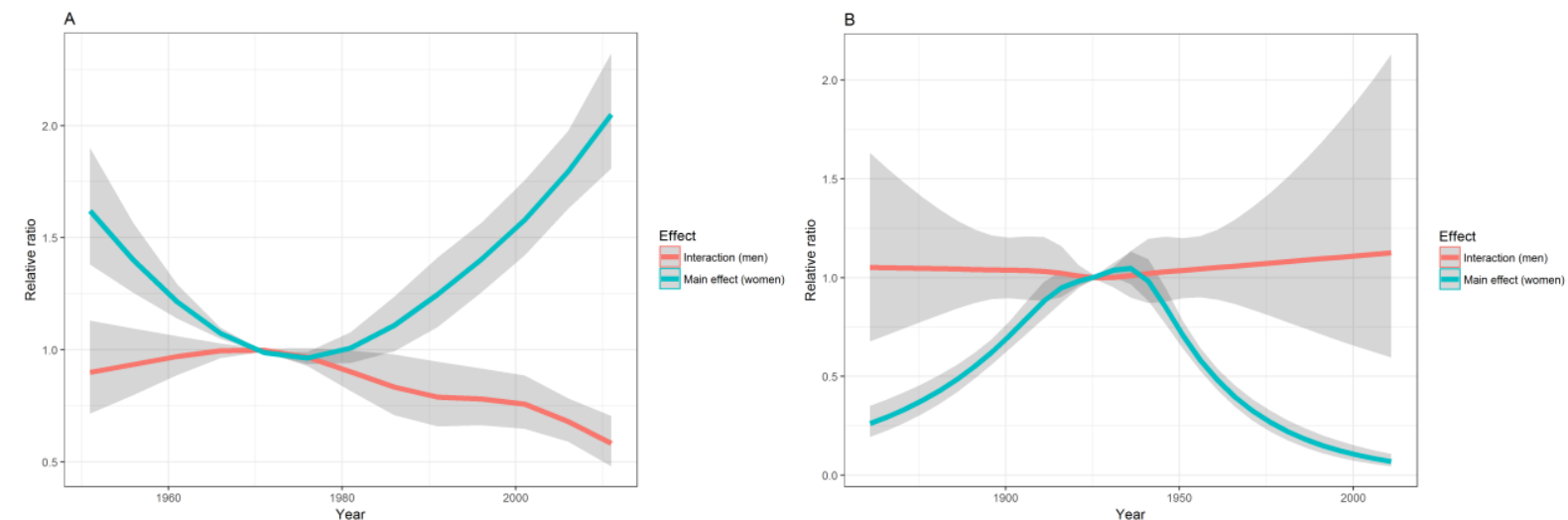

Fig.4 Period (A) -and cohort effects (B) for MS mortality data in Norway 1951-2015. Effects estimated from APC models with spline functions, with 1970 as reference period, 1925 as reference cohort, and linear time trend (also known as drift) assigned to period effect. The drift is consistent with the general increase in mortality observed both by year of death (period) and by year of birth (cohort), and should neither be attributed to period nor to cohort effects. Deviations from a linear trend indicate changes in period or cohort effects, respectively. The green line displays the main effect for females; the red line displays gender interaction (the relative male effect) with $95 \%$ CIs in grey.

\section{Discussion}

Recent epidemiological studies and reviews have emphasized possible cohort effects, suggesting that environmental factors explain both the "MS-epidemic" and the change in sex ratio [11-13]. In this study, we find stronger evidence for period effects driving an increasing female: male ratio since 1980, compatible with timedependent differences in case ascertainment between genders.

Life-style and environmental factors has changed dramatically for the cohorts represented in our dataset. The environmental risk factors underpinned by the strongest epidemiological evidence in MS are Epstein Barr virus 
infection, vitamin D insufficiency, overweight and smoking. Alterations in smoking habits, childhood obesity and vitamin D insufficiency have likely affected women more than men [14]. While we have insufficient historical data for the latter, "the obesity epidemic" among females in Norway started mainly after 1980 and would therefore have negligible effects on current mortality data $[15,16]$. Smoking was more than five times more common among men born 1890-1894, but a more pronounced and more prolonged increase among women had closed this gender gap in 1950 [17]. Unlike other candidate environmental risk factors, smoking increases MS risk regardless of age of exposure [18]. Smoke exposure is estimated to explain 20\% of MS cases in Sweden [19], and may indeed have contributed to our results. We would however expect a more gradual increase throughout the study period and a pronounced cohort effect up to 1950 if smoking was the main driver of the increasing MS mortality among women after 1980.

Period effects are typically associated with changes in diagnostic practice [20], which has obviously changed dramatically for the cohorts represented in our study period. Because people usually live many years with MS, factors contributing to altered diagnostic accuracy must be sought before the changes in MS associated mortality actually took place. Before world war II, it seems clear that MS was a particularly difficult disease to diagnose, and that is was easily misinterpreted as neurosyphilis or hysteria [21]. Until 1947 almost all MS-patients in Norway were diagnosed at the National Hospital in Oslo (named Kristiania until 1924) by one single professor of neurology [22]. The first department of neurology outside Oslo was established in Bergen in 1952. Hence, the barrier to an MS diagnosis must have been substantial, and was likely influenced by both proximity to the National Hospital, disease severity and social status. Males typically had greater income and higher social status. They are also relatively more frequently afflicted by primary progressive MS [23], which has a more disabling course than relapsing remitting MS (RRMS) [24], hence it may be speculated that men were less likely to escape diagnosis in earlier times when the diagnosis was almost totally dependent on clinical judgement. Among patients with conditions difficult to assess, women were more likely misdiagnosed with nosological categories such as hysteria [21].

Epidemiological research on MS emerged relatively late in Norway. The first study from 1952 does not specify gender [22], whereas two studies from the 1960ies reported a slight preponderance of females $[25,26]$. Between 1950 and 1979 neurological departments were established in most counties, facilitating more equal access to diagnosis. In parallel to the increasing female participation in the working force [27], Norway also implemented an extensive social security system in the late 60 -ies. Annuity payment requires a specific diagnosis, thus motivating more diagnostic accuracy among females. During the 70ies and 80ies, new technologies including electrophoresis and isoelectric focusing of immunoglobulin G, evoked potentials and MRI along with more sensitive diagnostic criteria also improved the diagnostic sensitivity and accuracy [28]. This was especially true for the more benign MS-forms, hence the increased MS incidence during the last decades are due to increase in RRMS only $[29,6]$. Indeed, studies on MS incidence trends report increasing sex ratio determined by people with RRMS [30,6,31].

The increase in MS associated mortality was most pronounced in older women, and the model best fitting our data also included the interaction between gender and age. Patients with RRMS outlives patients with primary progressive MS by almost 7 years [32]. The preferential increase in more benign RRMS-forms among women may therefore also explain the observed age-gradient in MS mortality. Gender differences in access or response to emerging treatment options for comorbid conditions, such as antibiotics that became available in the beginning of our study period, may potentially also have contributed to the observed gender-age interaction [33].

Immune modulating treatment aimed at RRMS has been available from mid 90ies. They could affect genders differently, either biologically or through motivation for diagnosis [33], potentially producing gender interactions with all three time effects. There is, however little data supporting substantial gender differences in therapeutic responses in MS [34]. Improved case ascertainment would cause period effects if equally distributed across age groups, and cohort effects if more pronounced in recent cohorts. Lastly, as for treatment against comorbid conditions, immune modulating treatment alters survival and hence changes age-effects. However, any effect of MS therapy would have restricted impact in this study because of the time gap between MS incidence and mortality. One potential exception could be progressive multifocal leukoencephalopathy or other lethal side 
effects occurring in an age group or a cohort at a time with few other MS associated deaths. Such fatal side effects are however very rare and has only occurred once in Norway.

In a recent study from Switzerland, both period and birth cohort effects contributed to the increasing preponderance of MS-associated mortality among women, and the cohort effect was emphasized [11]. In our study, the best fitting model did not include gender-cohort interaction. This does not exclude that cohort effects contributed, but they seem to be of minor importance. There are some notable differences in the temporal trends of MS mortality between Norway and Switzerland. Thus, MS risk peaked in cohorts born two decades earlier and gender-specific mortality diverged 5 decades earlier in Switzerland than in Norway [11]. The explanation to these marked differences could be related to environmental exposures, but also to the access to diagnostic services evolving differently in the two countries. Although the first neurological inpatient clinic was established later in Switzerland (1952) than in Norway (1895), neurological outpatient clinics appeared several decades earlier [35], as did also the first studies on MS epidemiology [11]. Notably, whereas the sex ratio increased until 2015 in our study, it has levelled out in Switzerland. Similar findings are observed for sex ratio of MS incidence in Norway, which has remained stable in cohorts born after 1930 [30]. In summary, these observations, along with our results, are compatible with a phase shifted decline in gender-specific barriers to MS diagnosis in the two countries.

There are limitations to this study. We were not able to validate against hospital files as these are very difficult to access for most of the study period. Notably, the gender ratio of death from amyotrophic lateral sclerosis (ALS) in Norway remained stable throughout the same period [36], arguing against a pronounced gender bias in access to neurological services. ALS is however a more characteristic and clinically homogenous disease than MS, it is almost always fatal and the delay between diagnosis and death is usually much shorter than for MS, the diagnosis is less dependent on laboratory investigations, and cannot to the same extent be confused with hysteria. Moreover, in previous studies from Western Norway spanning most of our study period, no gender difference has been observed when assessing mortality from MS between NCoDR and hospital files [32,37]. Gender variations in period effects are therefore more likely attributable to disproportional diagnostic accuracy between the genders than to biased registration in NCoDR. This is also in line with our sensitivity analysis, showing similar gender variation among those coded with MS as underlying cause of death as for all MS associated deaths.

Mortality data are restricted by lacking exhaustiveness in death registries as well as insufficient clinical data. In this study stratified analyses by MS type was not possible. Moreover, possible effects on more recent time trends in MS occurrence were unidentifiable because of the time gap between incidence and mortality. There is however some advantages in using mortality data; Registration in the NCoDR has been compulsory throughout the study period, and is therefore less likely affected by the possible sampling bias in studies based on disease registries, possibly explaining discordant results $[30,38,39]$. In Norway the importance of case ascertainment has been illustrated by earlier studies showing marked regional differences in MS occurrence $[40,22]$ which was not found in a recent study using compulsory nationwide registers [41].

In conclusion, age-period-cohort analyses of mortality data provide insights on historical time trends. For MS associated mortality in Norway, period effects best explain the emerging female preponderance during the last decades, compatible with a gradually more equitable access to diagnosis across the genders, in particular for more benign MS forms.

\section{Conflict of interest}

On behalf of all authors, the corresponding author declares that there is no conflict of interest.

\section{Ethical standards:}

The study was approved by the Regional Committee for Medical and Health Research Ethics (REC South East).

The manuscript does not contain clinical studies or individual patient data. 


\section{References}

1. Orton SM, Herrera BM, Yee IM, Valdar W, Ramagopalan SV, Sadovnick AD, Ebers GC (2006) Sex ratio of multiple sclerosis in Canada: a longitudinal study. The Lancet Neurology 5 (11):932-936. doi:10.1016/s1474-4422(06)70581-6

2. Alonso A, Hernan MA (2008) Temporal trends in the incidence of multiple sclerosis: a systematic review. Neurology 71 (2):129-135. doi:10.1212/01.wnl.0000316802.35974.34

3. Debouverie M, Pittion-Vouyovitch S, Louis S, Roederer T, Guillemin F (2007) Increasing incidence of multiple sclerosis among women in Lorraine, Eastern France. Multiple sclerosis (Houndmills, Basingstoke, England) 13 (8):962-967. doi:10.1177/1352458507077938

4. Westerlind H, Bostrom I, Stawiarz L, Landtblom AM, Almqvist C, Hillert J (2014) New data identify an increasing sex ratio of multiple sclerosis in Sweden. Multiple sclerosis (Houndmills, Basingstoke, England) 20 (12):1578-1583. doi:10.1177/1352458514530021

5. Heydarpour P, Mohammad K, Yekaninejad MS, Elhami SR, Khoshkish S, Sahraian MA (2014) Multiple sclerosis in Tehran, Iran: a joinpoint trend analysis. Multiple sclerosis (Houndmills, Basingstoke, England) 20 (4):512. doi:10.1177/1352458513494496

6. Celius EG, Smestad C (2009) Change in sex ratio, disease course and age at diagnosis in Oslo MS patients through seven decades. Acta Neurol Scand Suppl (189):27-29. doi:10.1111/j.16000404.2009.01208.x

7. Simpson S, Jr., Pittas F, van der Mei I, Blizzard L, Ponsonby AL, Taylor B (2011) Trends in the epidemiology of multiple sclerosis in Greater Hobart, Tasmania: 1951 to 2009. Journal of neurology, neurosurgery, and psychiatry 82 (2):180-187. doi:10.1136/jnnp.2010.215186

8. Holford TR (1991) Understanding the effects of age, period, and cohort on incidence and mortality rates. Annual review of public health 12:425-457. doi:10.1146/annurev.pu.12.050191.002233

9. Carstensen B (2007) Age-period-cohort models for the Lexis diagram. Statistics in medicine 26 (15):3018-3045. doi:10.1002/sim.2764

10. Akaike H (1974) A new look at the statistical model identification. IEEE Transactions on Automatic Control 19:716-723. doi:10.1109/TAC.1974.1100705

11. Ajdacic-Gross V, Schmid M, Mutsch M, Steinemann N, von Wyl V, Bopp M (2017) The change in the sex ratio in multiple sclerosis is driven by birth cohort effects. European journal of neurology : the official journal of the European Federation of Neurological Societies 24 (1):98-104.

doi:10.1111/ene.13160

12. Ajdacic-Gross V, Tschopp A, Schmid M, Bopp M, Gutzwiller F (2013) Missed epidemics and missing links: international birth cohort trends in multiple sclerosis. European journal of neurology : the official journal of the European Federation of Neurological Societies 20 (3):440-447.

doi:10.1111/j.1468-1331.2012.03802.x

13. Koch-Henriksen N, Sorensen PS (2010) The changing demographic pattern of multiple sclerosis epidemiology. The Lancet Neurology 9 (5):520-532. doi:10.1016/s1474-4422(10)70064-8

14. Ascherio A (2013) Environmental factors in multiple sclerosis. Expert review of neurotherapeutics 13 (12 Suppl):3-9. doi:10.1586/14737175.2013.865866

15. Meyer HE, Tverdal A (2005) Development of body weight in the Norwegian population. Prostaglandins, leukotrienes, and essential fatty acids 73 (1):3-7. doi:10.1016/j.plefa.2005.04.003 16. Brundtland GH, Liest $\varnothing \mid K$, Walløe L (1980) Height, weight and menarcheal age of Oslo schoolchildren during the last 60 years. Annals of Human Biology 7 (4):307-322.

doi:10.1080/03014468000004381

17. Ronneberg A, Lund KE, Hafstad A (1994) Lifetime smoking habits among Norwegian men and women born between 1890 and 1974. International journal of epidemiology 23 (2):267-276 
18. Hedstrom AK, Hillert J, Olsson T, Alfredsson L (2013) Smoking and multiple sclerosis susceptibility. European journal of epidemiology 28 (11):867-874. doi:10.1007/s10654-013-9853-4

19. Hedstrom AK, Olsson T, Alfredsson L (2016) Smoking is a major preventable risk factor for multiple sclerosis. Multiple sclerosis (Houndmills, Basingstoke, England) 22 (8):1021-1026.

doi:10.1177/1352458515609794

20. Rostgaard K, Vaeth M, Holst H, Madsen M, Lynge E (2001) Age-period-cohort modelling of breast cancer incidence in the Nordic countries. Statistics in medicine 20 (1):47-61

21. Talley CL (2005) The emergence of multiple sclerosis, 1870-1950: a puzzle of historical epidemiology. Perspectives in biology and medicine 48 (3):383-395. doi:10.1353/pbm.2005.0079

22. Swank RL, Lerstad O, Strom A, Backer J (1952) Multiple sclerosis in rural Norway its geographic and occupational incidence in relation to nutrition. The New England journal of medicine 246 (19):722-728

23. Miller DH, Leary SM (2007) Primary-progressive multiple sclerosis. The Lancet Neurology 6

(10):903-912. doi:https://doi.org/10.1016/S1474-4422(07)70243-0

24. Compston A, Coles A (2002) Multiple sclerosis. Lancet 359 (9313):1221-1231. doi:10.1016/s01406736(02)08220-x

25. Presthus J (1960) Report on the multiple sclerosis investigations in West-Norway. Acta

psychiatrica Scandinavica Supplementum 35 (147):88-92

26. Oftedal SI (1966) Multiple sclerosis in Vestfold, Norway. Acta neurologica Scandinavica 42:Suppl 19:19-26

27. Statistics Norway (1994) Historical statistics. http://www.ssb.no/a/histstat/tabeller/kap09.html. Accessed Dec 1st 2017

28. Trojano M (2011) Multiple sclerosis: the evolving diagnostic criteria for multiple sclerosis. Nature reviews Neurology 7 (5):251-252. doi:10.1038/nrneurol.2011.55

29. Midgard R, Riise T, Svanes C, Kvale G, Nyland H (1996) Incidence of multiple sclerosis in More and Romsdal, Norway from 1950 to 1991. An age-period-cohort analysis. Brain : a journal of neurology 119 ( Pt 1):203-211

30. Kampman MT, Aarseth JH, Grytten N, Benjaminsen E, Celius EG, Dahl OP, Holmoy T, LokenAmsrud K, Midgard R, Myhr KM, Risberg G, Vatne A, Torkildsen O (2013) Sex ratio of multiple sclerosis in persons born from 1930 to 1979 and its relation to latitude in Norway. Journal of neurology 260 (6):1481-1488. doi:10.1007/s00415-012-6814-x

31. Trojano M, Lucchese G, Graziano G, Taylor BV, Simpson S, Jr., Lepore V, Grand'maison F, Duquette $P$, Izquierdo G, Grammond P, Amato MP, Bergamaschi R, Giuliani G, Boz C, Hupperts R, Van Pesch V, Lechner-Scott J, Cristiano E, Fiol M, Oreja-Guevara C, Saladino ML, Verheul F, Slee M, Paolicelli D, Tortorella C, D'Onghia M, laffaldano P, Direnzo V, Butzkueven H (2012) Geographical variations in sex ratio trends over time in multiple sclerosis. PloS one 7 (10):e48078.

doi:10.1371/journal.pone.0048078

32. Lunde HMB, Assmus J, Myhr KM, Bo L, Grytten N (2017) Survival and cause of death in multiple sclerosis: a 60-year longitudinal population study. Journal of neurology, neurosurgery, and psychiatry 88 (8):621-625. doi:10.1136/jnnp-2016-315238

33. Franconi F, Campesi I (2014) Sex and gender influences on pharmacological response: an overview. Expert Review of Clinical Pharmacology 7 (4):469-485. doi:10.1586/17512433.2014.922866 34. Li R, Sun X, Shu Y, Mao Z, Xiao L, Qiu W, Lu Z, Hu X (2017) Sex differences in outcomes of diseasemodifying treatments for multiple sclerosis: A systematic review. Multiple sclerosis and related disorders 12:23-28. doi:10.1016/j.msard.2017.01.001

35. Bassetti C (2009) History of the Swiss Neurological Society in the context of the national and international development of neurology. vol 160. Schweizer Archiv für Neurologie und Psychiatrie 2009, 160(2):52-65.

36. Nakken O, Lindstrom JC, Tysnes OB, Holmoy T (2016) Mortality trends of amyotrophic lateral sclerosis in Norway 1951-2014: an age-period-cohort study. Journal of neurology 263 (12):2378-2385. doi:10.1007/s00415-016-8273-2 
37. Midgard R, Albrektsen G, Riise T, Kvale G, Nyland H (1995) Prognostic factors for survival in multiple sclerosis: a longitudinal, population based study in More and Romsdal, Norway. Journal of neurology, neurosurgery, and psychiatry 58 (4):417-421

38. Bostrom I, Stawiarz L, Landtblom AM (2013) Sex ratio of multiple sclerosis in the National Swedish MS Register (SMSreg). Multiple sclerosis (Houndmills, Basingstoke, England) 19 (1):46-52. doi:10.1177/1352458512446169

39. Trojano M (2014) The use of multiple population-based data sources for estimating MS sex ratio trends over time. Multiple sclerosis (Houndmills, Basingstoke, England) 20 (12):1551-1552.

doi:10.1177/1352458514536088

40. Midgard R (2012) Incidence and prevalence of multiple sclerosis in Norway. Acta Neurol Scand Suppl (195):36-42. doi:10.1111/ane.12025

41. Berg-Hansen P, Moen S, Harbo H, Celius E (2014) High prevalence and no latitude gradient of multiple sclerosis in Norway. Multiple sclerosis (Houndmills, Basingstoke, England) 20 (13):1780-1782. doi:10.1177/1352458514525871

\begin{tabular}{|c|c|c|c|c|}
\hline Model & Residual df $^{\mathrm{a}}$ & $\begin{array}{l}\text { Residual } \\
\text { deviance }\end{array}$ & Chi square & $\mathrm{AIC}^{\mathrm{b}}$ \\
\hline 1. $\mathrm{A}, \mathrm{P}, \mathrm{C}$ & 482 & 596.59 & & 2109 \\
\hline 2. A, P, C, S & 481 & 582.98 & $\mathrm{P}<0.001^{\mathrm{c}}$ & 2098 \\
\hline 3. A, P, C, S, AS & 477 & 575.13 & $\mathrm{P}=0.097^{\mathrm{d}}$ & 2098 \\
\hline 4. $\mathrm{A}, \mathrm{P}, \mathrm{C}, \mathrm{S}, \mathrm{PS}$ & 477 & 541.39 & $\mathrm{P}<0.001^{\mathrm{d}}$ & 2064 \\
\hline 5. A, P, C, S, CS & 477 & 540.38 & $\mathrm{P}<0.001^{\mathrm{d}}$ & 2063 \\
\hline 6. A, P, C, S, AS, CS & 473 & 531.95 & $\mathrm{P}=0.077^{\mathrm{e}}$ & 2063 \\
\hline 7. A, P, C, S, AS, PS & 473 & 526.87 & $\mathrm{P}=0.005^{\mathrm{f}}$ & 2057 \\
\hline 8. $\quad$ A, P, C, S, PS, CS & 473 & 530.86 & $\mathrm{P}=0.049^{\mathrm{e}}$ & 2061 \\
\hline 9. A, P, C, S, PS, CS, AS & 470 & 525.53 & $\mathrm{P}=0.097^{\mathrm{g}}$ & 2061 \\
\hline
\end{tabular}

Supplementary Table 1 Model fit for APC analysis for Norwegian MS mortality data 1951-2015. Main effects include age (A), period of death (P), birth cohort (C) and sex (S) listed in subsequently more complex models and including sex interaction effects. Goodness of fit for the different models can crudely be evaluated from the proximity between residual $\mathrm{df}$ and deviance. Chi square assessed by likelihood ratio tests between different nested models.

${ }^{\mathrm{a}}$ Degrees of freedom ${ }^{\mathrm{b}}$ Akaike's Information Criterion ${ }^{\mathrm{c}}$ As compared to model 1. ${ }^{\mathrm{d}}$ As compared to model 2. ${ }^{\mathrm{e}} \mathrm{As}$ compared to model $5 .{ }^{\mathrm{f}}$ As compared to model $4 .{ }^{\mathrm{g}}$ As compared to model 8 . 


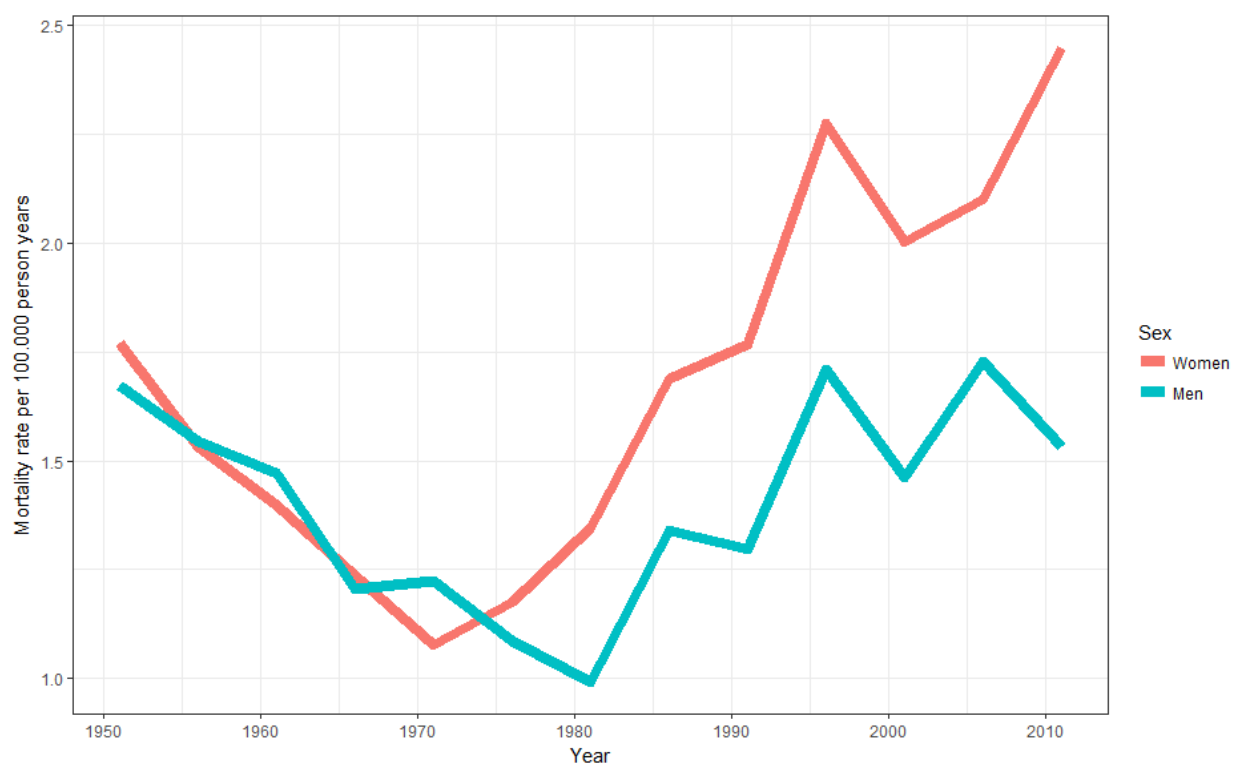

Supplementary Fig. 1. MS as underlying cause of death in Norway 1951-2015 by sex and period of death. Rates are standardized using the 2011-2015 Norwegian population as reference. A decreasing trend in both genders up until 1970 is caused by increasing differentiation into direct and contributing causes of death.
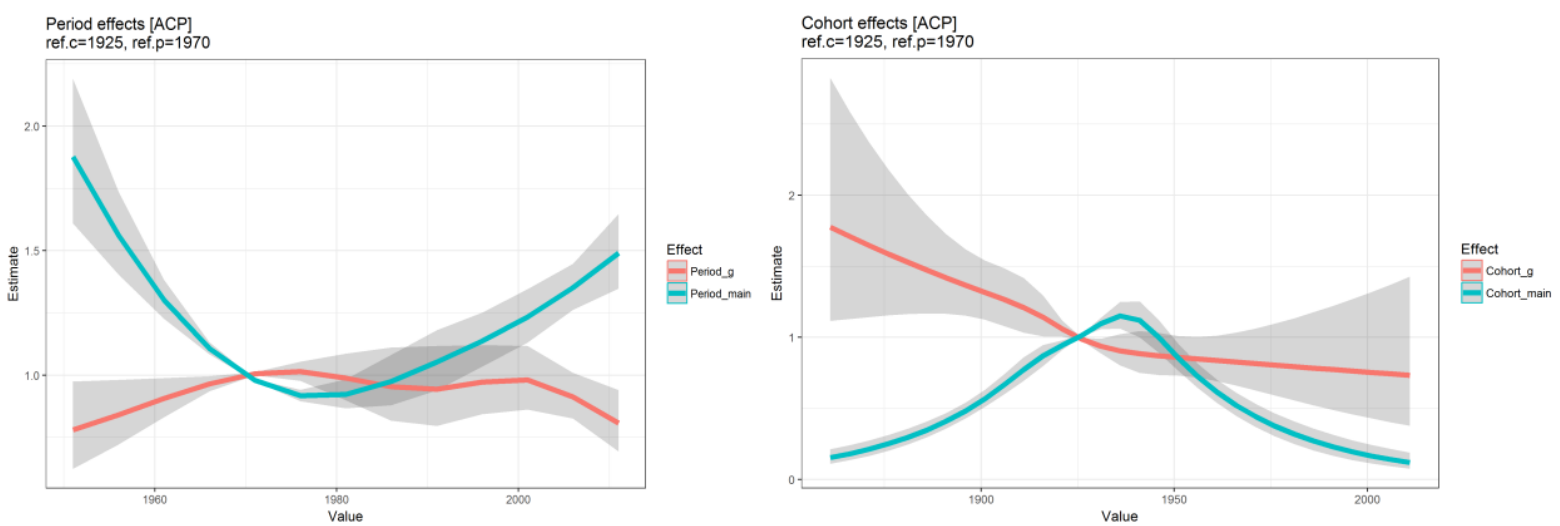

Supplementary Figure 2A and 2B Period (A) -and cohort effects (B) for MS mortality data in Norway 19512015. Effects estimated from APC models with spline functions, with 1970 as reference period, 1925 as reference cohort, and drift assigned to cohort effect. The green line displays the main effect for females; the red line displays gender interaction (the relative male effect) with 95\% CIs in grey. 